\title{
Hybridization of electromagnetic numerical methods through the $G$-matrix algorithm
}

\author{
J. P. Hugonin,* M. Besbes, and P. Lalanne \\ Laboratoire Charles Fabry de l'Institut d'Optique, CNRS, Université Paris-Sud, Campus Polytechnique, \\ RD 128, 91127 Palaiseau Cedex, France \\ *Corresponding author: jean-paul.hugonin@institutoptique.fr
}

Received March 12, 2008; revised June 11, 2008; accepted June 13, 2008; posted June 19, 2008 (Doc. ID 93801); published July 11, 2008

\begin{abstract}
For the sake of numerical performance, we hybridize two common approaches often used in electromagnetic computations, namely the finite-element method and the aperiodic Fourier modal method. To that end, we propose an extension of the classical $S$-matrix formalism to numerical situations, which requires handling different mathematical representations of the electromagnetic fields. As shown with a three-dimensional example, the proposed $G$-matrix formalism is stable and allows for an enhanced performance in terms of numerical accuracy and efficiency. (C) 2008 Optical Society of America

OCIS codes: $050.1755,050.1960,000.4430$.
\end{abstract}

With the undergoing development of micro- and nanotechnology, recent advances in microwave and photonic integrated circuits have increased the necessity of accurate computer-aided tools for solving Maxwell's equations. In this Letter, we are concerned with the hybridization of two popular frequencydomain numerical methods in electromagnetism: the finite-element method (FEM) and the aperiodic Fourier modal method (a-FMM). The former is a wellestablished method [1] operating in real space with space-variant discretizations. The latter is a generalization of the rigorous coupled-wave analysis (RCWA) [2] for analyzing nonperiodic geometries in the Fourier domain by using an artificial periodization with coordinate transforms [3]. Generally accepted appraisals of the two methods are different. The FEM is well appreciated for accurately handling intricate geometries, including sharp edges or curved discontinuities, but it is rather memory consuming for large three-dimensional (3D) meshes. By analytically handling one direction of space, the a-FMM relies only on a series of two-dimensional (2D) samplings and offers fully rigorous outgoing wave conditions in this direction, either for $z$-invariant [3] or periodic [4] waveguides. Drawbacks of the Fourier approach are difficulties encountered for analyzing metallic geometries with fields rapidly varying at subwavelength scales [5]. To take full advantage of their main strengths, the two methods can be combined. This can be achieved through a novel $G$-matrix formalism, a sort of generalization of the classical $S$-matrix formalism [6] to numerical situations mixing totally different mathematical representation of the electromagnetic fields.

To illustrate our purpose, let us consider Fig. 1. An aggregate of optical dielectric waveguides is illuminated by the fundamental Bloch mode of a periodic waveguide. Light is scattered by several inclusions, and notably by metallic scatterers depicted as vertical rices in the section $\left(z_{1}<z<\mathrm{z}_{2}\right)$. The whole scattering geometry can be rather large and may require a huge memory to be solved with methods relying on a full 3D discretization of space.
To overcome this numerical difficulty, the proposed hybridized approach (HYB) particularizes a specific space direction (the $z$ axis hereafter) for the integration of the differential systems of Maxwell's equations. The latter can be written as a single differential equation:

$$
\frac{\mathrm{d}}{\mathrm{d} z}[\boldsymbol{\Psi}]=D[\boldsymbol{\Psi}]
$$

with $[\Psi]$ a $n \times 1$ vector associated to the mathematical representation of the transverse electromagnetic field components, $E_{x} E_{y} H_{x} H_{y}$, in a local basis, and $D$ a differential operator that depends only on the $x, y$, and $z$ coordinates. The transverse fields can be expended into various basis: a Floquet Fourier-space basis with the RCWA [2], a local-mode basis with Fourier modal methods [3-5], a series of functions operating over a finite number of small subdomains with the FEM, etc. The choice of the basis depends on the local geometry. Solving the linear Maxwell's equations amounts to calculate the vectors $[\Psi(z)]$

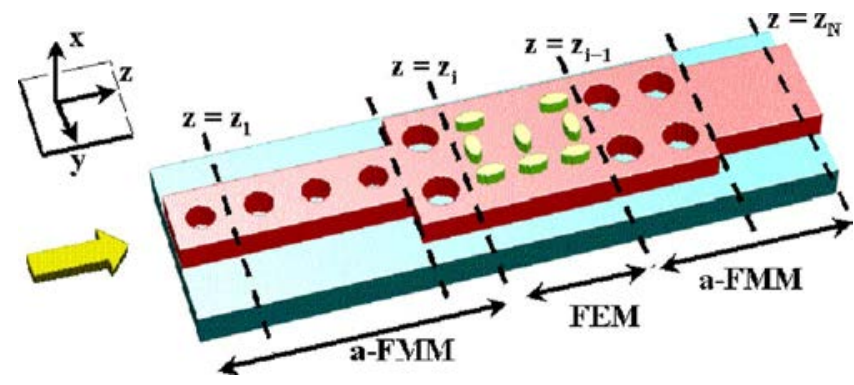

Fig. 1. (Color online) Sketch of a typical geometry considered for illustrating the relevancy of combining the FEM and the a-FMM. For $z_{i}<z<z_{i+1}$, the geometry encompasses metallic inclusions shown as vertical rices. The crosssection planes labeled $z=z_{i} i=1 \ldots \mathrm{N}$ represent cross-section planes related to G-matrix operations. At $z=z_{1}$ and $z=z_{N}$, modal field representations are used to satisfy the outgoing wave conditions in $z$-periodic and $z$-invariant waveguides, respectively. For computational purpose, complex coordinate transforms, in the transverse $x$ and $y$ directions, map infinite space to finite space. 
whose dimension may vary along the $z$ axis accordingly to the selected basis.

Two kinds of linear relations are obtained for $[\Psi(z)]$. First, the integration of Eq. (1) from plane $z$ $=z_{i}$ to plane $z=z_{i+1}$ leads to a linear relation between $\left[\boldsymbol{\Psi}\left(z_{i}\right)\right]$ and $\left[\boldsymbol{\Psi}\left(z_{i+1}\right)\right]$. This relation is analytically determined in the Fourier modal basis if $\varepsilon$ and $\mu$ are piecewise constant $[2,3]$. With the FEM, this relation is obtained by eliminating the inner finite-element (FE) nodes (in 2D) or edges (in 3D) from a large sparse matrix to retain only the FE degrees of freedom located on the $z_{i}$ and $z_{i+1}$ planes [7]. The second kind of relation is classically obtained by matching the tangential field components at a single plane $z=z_{i}$. It represents a basis transform that allows relating different mathematical representations of the same transverse electromagnetic field components in the modal, Fourier, or FE basis. The basis transform that allows matching different representations is particularly suitable to rigorously satisfy [4] the outgoing wave conditions at the outer planes $z=z_{1}$ and $z=z_{N}$. It is numerically performed in our numerical implementation by the method of moments; see, for instance, the earlier works $[8,9]$ in the present context of mixing Fourier and real-space discretization expansions. Without loss of generality, the two types of linear relations may be written in a compact form:

$$
\mathcal{L}[\boldsymbol{\Psi}]=\mathcal{R}\left[\boldsymbol{\Psi}^{\prime}\right],
$$

where $[\boldsymbol{\Psi}]$ and $\left[\boldsymbol{\Psi}^{\prime}\right]$ are $n \times 1$ and $n^{\prime} \times 1$ generic vector notations representing the transverse field components, either in two different cross-section planes $\left(z\right.$ and $\left.z^{\prime}\right)$ or in two different basis at the same crosssection plane $z$, or in both different basis and different cross-section planes. In Eq. (2), $\mathfrak{L}$ and $\mathfrak{R}$ are two matrices with an identical number $m$ of lines. Note that the $m \times n \mathfrak{L}$ and $m \times n^{\prime} \mathfrak{R}$ matrices are rectangular and cannot be inverted in general.

Referring to Fig. 1, a set of equations similar to Eq. (2) may be written for every plane $z_{i}$. Doing so, we would end up with a huge linear system whose unknowns are the $\left[\Psi\left(z_{i}\right)\right]$ 's. If the problem is well conditioned, the system can be solved by eliminating the intermediate unknowns, and one may compute the important unknowns (for example, the diffracted fields at $z=z_{1}$ and $z_{N}$ ) as a function of the incident fields. Although very stable and efficient algorithms (such as the Gauss-Jordan elimination algorithm [10]) may be used for the elimination, this brute-force approach would necessitate handling huge matrices. As we will see, the Gauss-Jordan elimination can be efficiently carried out, step by step.

For that purpose, let us now consider another linear relationship between $\left[\boldsymbol{\Psi}^{\prime}\right]$ and another $n^{\prime \prime} \times 1$ vector $\left[\boldsymbol{\Psi}^{\prime \prime}\right]$. The two relations relating the three field representations, $[\boldsymbol{\Psi}],\left[\boldsymbol{\Psi}^{\prime}\right]$, and $\left[\boldsymbol{\Psi}^{\prime \prime}\right]$, can be written as

$$
\begin{array}{r}
\mathcal{L}[\boldsymbol{\Psi}]-\mathcal{R}\left[\boldsymbol{\Psi}^{\prime}\right]=[\mathbf{0}], \\
\mathcal{L}^{\prime}\left[\boldsymbol{\Psi}^{\prime}\right]-\mathcal{R}^{\prime}\left[\boldsymbol{\Psi}^{\prime \prime}\right]=[\mathbf{0}] .
\end{array}
$$

For $m+m^{\prime} \geqslant n^{\prime}$, one can eliminate [ $\left.\boldsymbol{\Psi}^{\prime}\right]$ from the augmented system of Eqs. (3a) and (3b) to obtain a new linear relation between $[\boldsymbol{\Psi}]$ and $\left[\boldsymbol{\Psi}^{\prime \prime}\right]$ :

$$
\mathcal{L}^{\prime \prime}[\boldsymbol{\Psi}]=\mathcal{R}^{\prime \prime}\left[\boldsymbol{\Psi}^{\prime \prime}\right] \text {. }
$$

Clearly, for each elimination step, it is necessary to introduce as many new relations as eliminated unknowns, so that the number $m^{\prime \prime}$ of lines in $\mathfrak{L}^{\prime \prime}$ and $\mathfrak{R}^{\prime \prime}$ is equal to $m+m^{\prime}-n^{\prime}$. Symbolically, if we denote by $\mathbf{G}$ the matrix pair $(\mathfrak{L}, \mathfrak{R})$ involved in the linear relations, the elimination operation $\mathbf{G}^{\prime \prime}=\mathbf{G}^{\prime} \cdot \mathbf{G}$ leading to Eq. (4) defines an inner operation that we call "product" hereafter, in a similar spirit as the $S$-matrix algorithm defines a "product." However, let us note that the $S$-matrix product involves several matrix multiplications and inversions, while the $G$-matrix product does not rely on any matrix algebra and is performed fully numerically, thanks to the efficient Gauss-Jordan elimination algorithm [10]. Thus referring to Fig. 1, the solution of the scattering problem can be obtained by using $G$-matrix products to repeatedly eliminate the unknowns in the intermediate planes, $z_{2}, z_{3} \ldots z_{N-1}$, and to obtain a $\mathbf{G}$ matrix $\mathbf{G}=(\mathfrak{L}, \mathfrak{R})=\mathbf{G}_{\mathrm{N}} \ldots \mathbf{G}_{2} \cdot \mathbf{G}_{1}$ that relates the amplitude vectors of the incident $\mathbf{I}$ and diffracted $\mathbf{D}$ waves in the modal basis at the outer planes $z_{1}$ and $z_{N}$ : $\mathfrak{L}\left[\begin{array}{c}\mathbf{I}_{1} \\ \mathbf{D}_{1}\end{array}\right]=\mathfrak{R}\left[\begin{array}{c}\mathbf{I}_{N} \\ \mathbf{D}_{N}\end{array}\right]$.

It is interesting to consider the particular case where all the transverse field vectors $[\Psi]$ have the same lengths $\left(n=n^{\prime}\right)$. Then the $G$ matrix $\mathbf{G}=(\mathfrak{L}, \mathfrak{R})$ can be simply related to the classical $S$ matrix by

$$
\mathcal{L}=\left[\begin{array}{cc}
\mathbf{S}_{11} & \mathbf{0} \\
\mathbf{S}_{21} & -\mathbf{I}
\end{array}\right], \quad \mathcal{R}=\left[\begin{array}{cc}
\mathbf{I} & -\mathbf{S}_{12} \\
\mathbf{0} & -\mathbf{S}_{22}
\end{array}\right],
$$

where $\mathbf{S}_{11}, \mathbf{S}_{12}, \mathbf{S}_{21}$, and $\mathbf{S}_{22}$ are the four submatrices [6] of the $\mathbf{S}$ matrix that relates modal expansions. Although it does not involve any matrix algebra manipulations, the G-matrix product becomes equivalent to the classical S-matrix product. Equation (4) has been previously used for Bloch mode calculations with the a-FMM [11] and with the FEM [8]. For large matrices, the CPU time required for performing a $G$-matrix product is slightly longer than that of an $S$-matrix product, but it is more accurate and more stable, and since the $S$-matrix algorithm cannot be used if the transverse field vector lengths vary, the G-matrix elimination algorithm can be considered as a generalization of the classical S-matrix algorithm. As evidenced in the following numerical results, the G-matrix elimination algorithm is numerically stable, and the HYB approach is efficient even for dielectric structures.

We have tested the HYB performance for various scattering constructs involving metallic and dielectric materials. Recently, the HYB was convincingly benchmarked [12] against 11 other methods for a $2 \mathrm{D}$ metallic slit-groove lamellar diffraction problem. Here, we provide a 3D example and compute the even gap-guided mode of a photonic-crystal single-rowmissing W1 waveguide in a semiconductor $(n=3.5)$ membrane in air. The Bloch mode computation is 
performed for a frequency $(\lambda=1.55 \mu \mathrm{m})$ corresponding to a null group velocity at the boundary of the Brillouin zone [13]. In Fig. 2, we compare the numerical results obtained with the a-FMM (a) and HYB (b) methods for the ratio $\left|\mathbf{E}_{A}\right| /\left|\mathbf{E}_{B}\right|$ of the $z$ components of electric-field modulus at points A and B (right inset) located in the median plane of the membrane. For the calculations, the integration of the differential Maxwell's equations is performed along the $z$ direction, which is transverse to the waveguide axis (right inset). Details of the a-FMM implementation for this specific geometry can be found in [14]. With the a-FMM, the continuous profile is actually discretized with a staircase approximation (left inset), and the accuracy depends on the number $S$ of slides used to discretize the nearest hole rows surrounding the line defect. As shown in Fig. 2(a), a plateau is systematically obtained as the total number $N$ of Fourier harmonics increases, but the convergence with $S$ is rather small even for $S=79$ slices $(\asymp \lambda / 500)$ [5]. This drawback is completely removed with the HYB method [Fig. 2(b)], which uses a nonuniform tetrahedron mesh with first-order elements. Because of the accurate sampling in real space, convergence is guaranteed as the number $N^{\prime}$ of nonuniform mesh edges increases. More specifically, it is found that the HYB

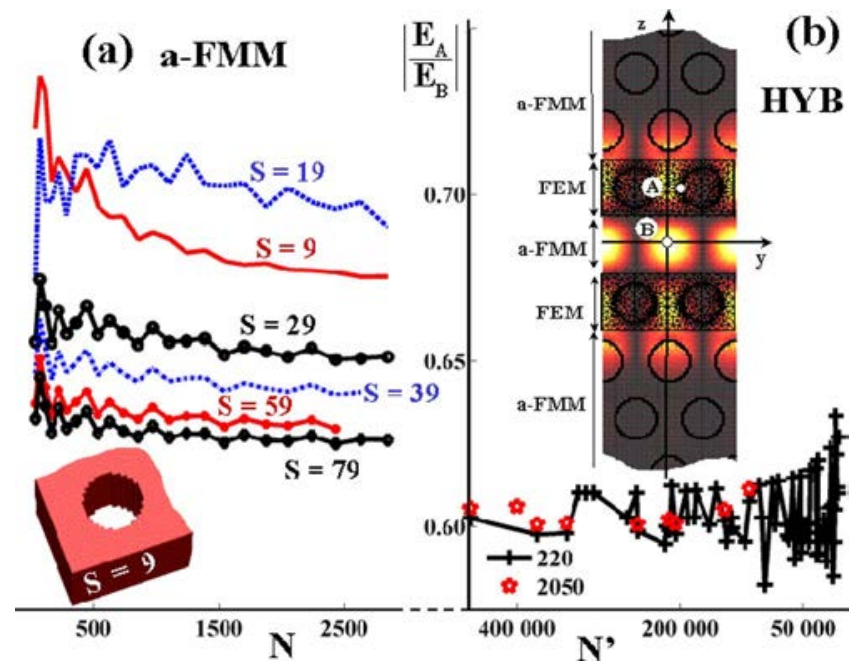

Fig. 2. (Color online) 3D field calculation of the gap-guided mode of a W1 waveguide in a semiconductor $(n=3.5)$ membrane in air with the (a) a-FMM and (b) HYB methods. The vertical axis represents the ratio $\left|\mathbf{E}_{A}\right| /\left|\mathbf{E}_{B}\right|$ of the $z$-electricfield modulus at points $A$ and $B$ (right inset) located in the median plane of the membrane. $\left|\mathbf{E}_{A}\right| /\left|\mathbf{E}_{B}\right|$ is plotted as a function of the total number $N$ of Fourier harmonics in (a) for different slicing numbers $S$ of the two inner rows (9 slices being used for the other hole rows). In (b), $\left|\mathbf{E}_{A}\right| /\left|\mathbf{E}_{B}\right|$ is plotted as a function of the total number of elements used to discretize the inner first rows for two values of $N(N$ $=220$ and 2050). The results hold for a period $a=420 \mathrm{~nm}$, a membrane thickness $t=220 \mathrm{~nm}$, and a hole radius $r=0.3 a$. The wavelength is $\lambda=1.55 \mu \mathrm{m}$. predictions for $N^{\prime}=50,000$ and $N=220$ are more accurate than those of the a-FMM for $N$ as large as 2500 . This corresponds to an impressive lowering of memory requirements and CPU times for a given accuracy.

Since it relies on classical and direct numerical procedures, we believe that the present $G$-matrix formalism can be applied to a broad variety of numerical methods relying on linear differential operators. For instance, it can be applied to FEM and FMM individually or to their natural combination for the sake of performance improvement. Besides, this approach might be important for coupling multiphysics problems.

This research is supported under the European contract SPLASH of the Sixth EU Framework programme and by the Agence Nationale de la Recherche under contract MIRAMAN of the French ANR Nano2006.

\section{References and Notes}

1. J. Jin, The Finite Element Method in Electromagnetics, 2nd ed. (Wiley, 2002).

2. M. G. Moharam, E. B. Grann, D. A. Pommet, and T. K. Gaylord, J. Opt. Soc. Am. A 12, 1068 (1995).

3. J. P. Hugonin and P. Lalanne, J. Opt. Soc. Am. A 22, 1844 (2005).

4. G. Lecamp, J. P. Hugonin, and P. Lalanne, Opt. Express 15, 11042 (2007).

5. E. Popov, M. Nevière, B. Gralak, and G. Tayeb, J. Opt. Soc. Am. A 19, 33 (2002).

6. H. Kim, I. M. Lee, and B. Lee, J. Opt. Soc. Am. A 24, 2313 (2007).

7. K. Dossou, M. A. Byrne, and L. C. Botten, J. Comput. Phys. 219, 120 (2006).

8. T. Delort and D. Maystre, J. Opt. Soc. Am. A 10, 2592 (1993).

9. P. Lalanne and J. P. Hugonin, J. Opt. Soc. Am. A 17, 1033 (2000).

10. With the MATLAB software, we have implemented several methods using LU and QR factorizations for the Gauss-Jordan elimination; see W. H. Press, B. P. Flannery, S. A. Teukolsky, and W. T. Vetterling, Numerical Recipes: The Art of Scientific Computing (Cambridge U. Press, 1989), Chap. 3.

11. Q. Cao, P. Lalanne, and J. P. Hugonin, J. Opt. Soc. Am. A 19, 335 (2002).

12. M. Besbes, J. P. Hugonin, P. Lalanne, S. van Haver, O. T. A. Janssen, A. M. Nugrowati, M. Xu, S. F. Pereira, H. P. Urbach, A. S. van de Nes, P. Bienstman, G. Granet, A. Moreau, S. Helfert, M. Sukharev, T. Seideman, F. I. Baida, B. Guizal, and D. Van Labeke, J. Eur. Opt. Soc. Rapid Publ. 2, 07022 (2007).

13. We note that accurately calculating the W1 modal field on the hole discontinuities is crucial for estimating the inevitable extrinsic loss in the slow-light regime, see L. C. Andreani and D. Gerace, Phys. Status Solidi B 244, 3528 (2007).

14. C. Sauvan, P. Lalanne, J. C. Rodier, J. P. Hugonin, and A. Talneau, IEEE Photonics Technol. Lett. 15, 1243 (2003). 